\title{
"It is the only constant in what feels like a completely upside down and scary world": Living with an eating disorder during COVID-19 and the importance of perceived control for recovery and relapse
}

\author{
Dawn Branley-Bell ${ }^{\mathrm{a}, *}$, Catherine V. Talbot ${ }^{\mathrm{b}}$ \\ ${ }^{\text {a }}$ Department of Psychology, Northumbria University, Newcastle Upon Tyne, England, UK \\ ${ }^{\mathrm{b}}$ Department of Psychology, Faculty of Science and Technology, Bournemouth University, England, UK
}

\section{A R T I C L E I N F O}

\section{Keywords:}

COVID-19

Long-term care

Feeding and eating disorders

Coronavirus

Anorexia nervosa

Bulimia nervosa

Eating behavior

\begin{abstract}
A B S T R A C T
The COVID-19 pandemic has had a profound, negative impact on the lives and wellbeing of much of the population, and it can raise additional challenges for individuals with eating disorders (EDs). During early stages of the UK lockdown, individuals reported disruptions to many aspects of their lives, including reduced feelings of control and serious concerns over the impact of the pandemic on ED symptoms and/or recovery. This study applied a mixed methods online survey to collect responses from 58 individuals (age 16-65yrs) with lived experience of EDs. Data was collected across two time points (April 2020 and June 2020) to explore the ongoing impacts of the pandemic on this population. The results suggest that higher perceptions of general, external control may be associated with ED recovery. Quantitative results show that individuals who reported recovering from their ED since the first time point, also reported significant increases in perceived control (compared to individuals who had relapsed or whose ED status was unchanged). Thematic analysis generated two themes: ED behaviours as an 'auxiliary control mechanism', and loss of auxiliary control after lockdown. Individuals who experienced less perceived control reported a tendency to rely upon eating disorder behaviours as an auxiliary coping mechanism, i.e., diminished external control was directed inwards and replaced with controlling their own behaviour. The preliminary results suggest that perceived control may be a significant factor in ED recovery. Individuals with EDs may be at significant risk of detrimental impacts on their recovery and wellbeing because of the pandemic reducing peoples' sense of control. These preliminary findings highlight the need for further research in this area, including investigation around potential interventions based upon strengthening perceptions of control to promote ED recovery.
\end{abstract}

\section{Background}

Research shows that the COVID-19 pandemic has had a profound, negative impact on the lives of many people with eating disorders (EDs). This has been linked to numerous factors including increased media exposure, disruption to daily activities, social isolation, modified physical activity and sleep, negative affect and fear of contagion (Branley-Bell \& Talbot, 2020; Rodgers et al., 2020; Talbot \& Branley-Bell, 2021). Recent empirical research from Australia (Phillipou et al., 2020), the United Kingdom (UK; Branley-Bell \& Talbot, 2020; Vuillier et al., 2021), the Netherlands and United States (Termorshuizen et al., 2020) reports evidence of increased ED behaviours during the early stages of the pandemic. For example, Phillipou et al. (2020) report increased restricting, binge eating, purging, and exercise behaviours among people with history of EDs; Termorshuizen et al. (2020) report increased food restriction among people with anorexia nervosa and increased binge-eating episodes for individuals with bulimia nervosa and binge-eating disorder; Branley-Bell and Talbot (2020) found people with EDs reported worsened symptoms, changes to physical activity rates and detrimental relationships with food. It is worth highlighting that for some individuals, the pandemic has also had positive impacts, for example Branley-Bell and Talbot (2020) found that a minority of participants reported positive changes to their living situation, engaging in fewer social comparisons, and wearing less 'triggering' clothing.

Whilst these findings provide valuable insight into the impact of the pandemic on people with EDs, they tend to rely upon a single point of

\footnotetext{
* Corresponding author.

E-mail address: dawn.branley-bell@northumbria.ac.uk (D. Branley-Bell).
} 
data collection representing a solo 'snapshot' in time. To date, little is known about the impacts of the prolonged pandemic and continued lockdown periods on people with EDs. Longitudinal research is required to track their experiences of the pandemic and its impact upon symptoms and wellbeing. The current study aims to investigate the ongoing effects of the pandemic between two time points (first, soon after the start of the UK lockdown, April 2020, and second as the first lockdown restrictions began to ease, June 2020). A mixed-methods online survey was used, including established quantitative measures to investigate five factors related to wellbeing during the pandemic: mental wellbeing, perceived stress, social support, perceived control, and ED rumination, and open-ended questions to gather qualitative data. The five quantitative factors were chosen due to their established links with ED aetiology. Decreased mental health (Doll et al., 2005), low perceived control (Bruch, 2001) and high rumination (Rawal et al., 2010) have been linked to increased ED symptoms. Whilst positive social support has been shown to play a protective role, i.e., helping to decrease symptoms (Marcos \& Cantero, 2009).

The COVID-19 pandemic has already been linked to decreased mental wellbeing and increased stress for both individuals with mental health issues (Yao et al., 2020) and the wider population (Pfefferbaum \& North, 2020; Rodríguez-Rey, 2020; Usher et al., 2020). For individuals experiencing - or in recovery from - an ED, prolonged periods of lockdown have the potential to impact upon access to social support and increase ED rumination (Branley-Bell \& Talbot, 2020), which could potentially lead to an increase in ED behaviour.

It is likely that many of the population have felt a reduced sense of control about many aspects of their lives as a result of the pandemic (Hou et al., 2020). Control is thought to play a key role in the aetiology and maintenance of EDs (Froreich et al., 2016). Anorexia nervosa, for example, is associated with low levels of openness to unexpected events and avoidance of uncertainty (Hempel et al., 2018). Consistent with this, researchers have found that people with EDs experienced a loss of control at the start of the pandemic; consequently, turning to ED behaviours such as food-restriction to regain some sense of control (Branley-Bell \& Talbot, 2020; McCombie et al., 2020; Richardson et al., 2020; Schlegl et al., 2020).

Of course, sense of control is not only important to individuals with EDs, but to the population more widely. A recent study in China by Zheng et al. (2020) indicates that increased perceptions of control can act as a protective mechanism against psychological distress during a pandemic. Their results suggest that individuals with higher levels of perceived control also perceived themselves to be more psychologically distanced from the disease, helping them to cope better with the stresses of the pandemic. Zheng et al. suggest that research into the role of control for other populations and cultures would be beneficial. We therefore investigate whether control played a protective role for UK individuals with experience of EDs during the first few months of the UK pandemic.

The purpose of our study was to investigate the longitudinal impacts of the pandemic on people with EDs. We hypothesised that eating disorder status, mental wellbeing, perceived stress, social support, perceived control, and ED rumination would be negatively impacted between April 2020 and June 2020.

\section{Method}

Ethical approval was granted by Northumbria University ethics committee [23259] prior to data collection and all participants provided informed consent. Participants were recruited via Twitter and Facebook using opportunistic and snowball sampling. Inclusion criteria were as follows: Over 16 years of age, UK resident with self-reported experience of an ED (including those in recovery). Recruitment was limited to the UK to allow accurate identification of the stage of the pandemic and to ensure that data was comparable in this regard.

Participants in this study completed two anonymous online surveys about their experiences of the pandemic. The first survey took place two weeks after lockdown restrictions were first enforced in the UK (April 2020), and the follow-up 8-10 weeks later when the first lockdown restrictions started to ease (June 2020). Both surveys included established scales linked to ED psychopathology (see measures section), as well as closed and open-ended questions about symptoms, living situation, social isolation, long-lasting impacts of the pandemic, media messages, physical activity, online activities, and coping mechanisms. All participants were debriefed following completion of each survey. 153 individuals enrolled to take part in the first survey, however 24 participants were excluded resulting in a sample of 129 participants at time point 1. Participants were then e-mailed an invitation to participate in the second survey in June 2020. Of these, 58 participated at the second time point, the data for which is used in this paper (Fig. 1).

\subsection{Measures}

Established scales were included to measure five key factors linked to ED psychopathology: mental wellbeing (Doll et al., 2005), perceived stress (Hou et al., 2013), social support (Ghaderi, 2003), perceived control (Dalgleish et al., 2001), and ED rumination (Rawal et al., 2010). This enabled the researchers to test for any significant changes in these factors as lockdown progressed.

\subsubsection{Warwick-Edinburgh mental wellbeing scale (SWEMWBS)}

The 7-item SWEMWBS, was used to measure emotional and functioning aspects of mental wellbeing (NHS Health Scotland, 2016; Tennant et al., 2007). The measure asks individuals how often over the past two weeks they have felt: optimistic about the future; useful; relaxed; and how often they have been: dealing with problems well; thinking clearly; feeling close to other people; and able to make up their own mind about things. An overall mental wellbeing score is calculated, ranging from 7 to 35, with higher scores indicative of greater mental wellbeing. Cronbach's alpha for this scale in the current study = 0.84-0.88.

\subsubsection{Perceived stress scale (PSS-4)}

The PSS is a self-report measure of the degree to which an individual perceives situations in their life as stressful, and it is one of the most widely used psychological measures of stress (Cohen et al., 1983). Respondents are asked to evaluate the degree to which they believe their life has been unpredictable, uncontrollable, and overloaded. In this study, the shortened 4-item version (PSS-4) was used. The 4-item version has been widely disseminated and has been shown to be a useful instrument for assessing stress perception levels across many populations (Vallejo et al., 2018), with good internal consistency and reliability (Mitchell et al., 2008). The PSS-4 gives a final score out of 16, with higher scores indicating greater perceived stress. Cronbach's alpha for this scale in the current study $=0.73-0.77$.

\subsubsection{Enrichd social support inventory (ESSI)}

Poor social support has been identified as a risk factor for ED onset (Ghaderi, 2003). The ESSI is a brief 7-item scale designed to measure the existence or availability of people on who an individual can rely (Vaglio et al., 2004). The individual items are summed to create a total score, between 8 and 34, with higher scores indicating greater social support. Cronbach's alpha for this scale in the current study $=0.81-0.84$.

\subsubsection{Shapiro control inventory (SCI-general)}

Perceived control over external events has been linked to the onset and maintenance of ED psychopathology (Dalgleish et al., 2001). In fact, some suggest that a need for general self-control is often present prior to its manifestation as control over eating behaviours (Fairburn et al., 1999). The SCI general domain scale (Shapiro, 1994) is a 16-item scale encompassing positive and negative sense of control. The SCI-general gives a score between 16 and 112, with higher scores indicating 


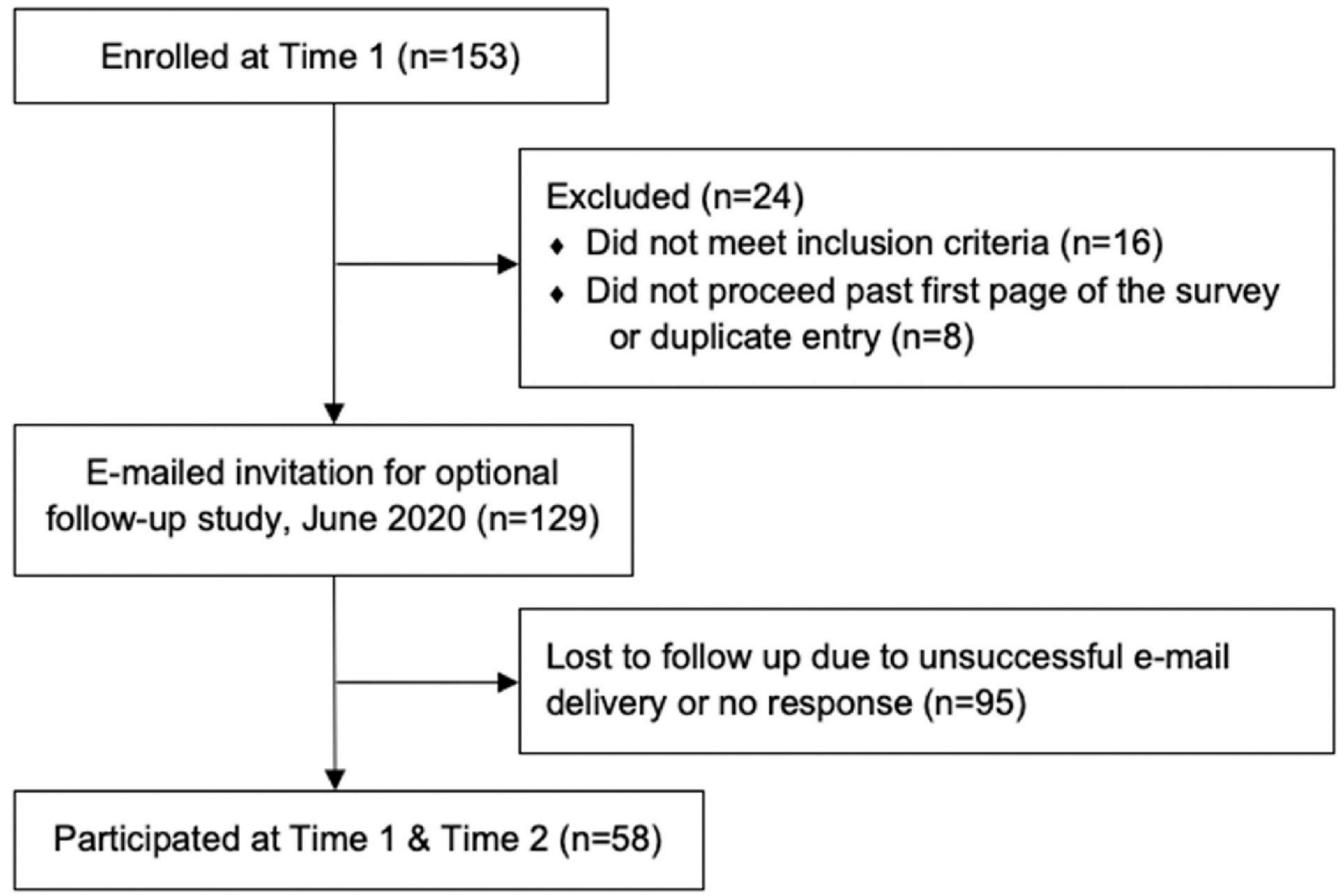

Fig. 1. Recruitment and sample numbers at each time point.

greater perceptions of control. The scale has been described as a useful instrument for studying control in relation to EDs (Lee et al., 2005). Cronbach's alpha for this scale in the current study $=0.87-0.90$.

\subsubsection{Ruminative response scale for eating disorders (RRS-ED)}

Rumination refers to a cognitive process involving repetitive negative thoughts. Ruminative thoughts have been heavily linked to eating disorder psychopathology (Rawal et al., 2010; Smith et al., 2018) and is regarded as a key factor in ED maintenance (Cowdrey \& Park, 2012; Park et al., 2011). The RRS-ED is the first ED-specific rumination measure. Using 9-items, the measure gives a total score between 9 and 36, with higher scores indicative of greater ruminative thoughts. Cronbach's alpha for this scale in the current study $=0.84-0.89$.

\subsubsection{Open-ended questions}

Open-ended questions included asking individuals about: changes to living situations since the start of the pandemic, changes to feelings of social isolation, the type of media they have seen about the pandemic, the impact of the pandemic on their ED symptoms so far, any changes to their coping mechanisms since the pandemic, and any foreseen longerterm impacts of the pandemic in relation to their ED (see Supplementary Material).

\subsection{Analytical approach}

A mixed methods approach was applied. Quantitative data were analysed using SPSS v.26. Before analysis, data were tested for possible violations of the assumptions of normality and no multicollinearity. One extreme outlier on wellbeing score was identified (Z-score $>3$ ), this score was excluded from the data. All other data points met normality assumptions. Changes in self-reported ED status between time point 1 and time point 2 were identified and participants were subsequently grouped into those who were: relapsing, recovering, or experiencing no change in ED status. A series of 2 (Time) x 3 (Group) mixed ANOVAS were then calculated to compare the three groups across wellbeing, enriched social support, ED rumination, perceived stress, and perceived control.

Quantitative analyses were complemented with a thematic analysis (using NVivo) of open-ended responses (Braun \& Clarke, 2006) that specifically explored the impact of perceived control on respondents' ED symptoms and wellbeing. The data were searched for responses relating to control, coded, and subsequently grouped into categories. Categories were developed into themes, with both authors agreeing on the finalised versions.

\section{Results}

\subsection{Sample demographics}

58 participants completed both the initial survey and the follow-up survey. Participants were aged between 16 and 65 years $(M=30.86$ yrs, $S D=11.12$ yrs), with 57 identifying as female $(98.3 \%)$ and one as male $(1.7 \%)$. The majority ( $\mathrm{n}=37,63.8 \%$ ) of the sample described themselves as currently experiencing an ED (or in a period of relapse from recovery), with the remaining 21 (36.2\%) describing their status as in recovery. Of those 21 participants in recovery, 4 (19\%) reported being in recovery for less than 3 months, $3(14.3 \%)$ participants had been in recovery for 3-12 months, and 14 (66.7\%) for over 1 year).

Twenty-eight of the participants (48.3\%) identified themselves as having anorexia nervosa, $7(12.1 \%)$ bulimia nervosa, 3 (5.3\%) Other Specified Feeding and Eating Disorder, 2 (3.5\%) 1 (1.7\%) binge eating disorder, and $12(20.7 \%)$ reported symptoms of multiple EDs. Seven participants (12.1\%) chose not to reveal their specific ED. 


\subsection{ED recovery and relapse during lockdown: Quantitative analysis}

Bivariate correlations (Table 1) were explored prior to the ANOVA analysis. At both time points, perceived control was negatively correlated with perceived stress and ED rumination, and positively correlated with mental wellbeing (the latter was also negatively correlated with stress at both time points, and rumination at time point 1 ).

At the second time point, perceived stress was positively correlated with ED rumination and negatively correlated with social support and, as aforementioned, perceived control.

The ED status for some of the participants changed between time point 1 and time point 2, with 9 (15.5\%) participants relapsing during this time, 11 (19\%) participants recovering, and the remaining 38 (65.5\%) reporting no change in their ED status.

Comparing the three groups (stable, recovery, relapse) using a series of 2 (Time) x 3 (Group) mixed ANOVAs showed no significant between or within group effects for 4 of the variables: wellbeing, enriched social support, ED rumination and perceived stress.

However, some interesting results were found for perceived control. The main effect for group was not significant $(F(2,55)=3.02, \mathrm{p}=.06$, $\eta_{\mathrm{p}}^{2}=0.10$ ) with similar control scores for the recovery group, relapse group and the stable/no change group at each time point. There was also no significant main effect of time, $F(1,55)=3.42, p=.07, \eta_{p}^{2}=0.06$, with control scores at both time points remaining similar.

However, there was significant interaction effect between time and group, $F(2,55)=4.80, p=.01$, and this was a large effect $\left(\eta_{\mathrm{p}}^{2}=0.15\right)$. The interaction was investigated using post-hoc paired t-tests using a Bonferroni correction for multiple comparisons $(\alpha=0.05 / 3=0.017)$. The results show a significant increase in control scores for individuals in the recovery group from Time 1 to Time $2, t(10)=-4.01, p=.002$. In comparison, there was no significant difference between the time points for individuals in the stable group, $t(37)=0.562, p=.577$; or the relapse group, $t(8)=-0.166, p=.873)$. Mean scores and standard deviations for all measures are shown in Table 2.

This interaction can also be clearly seen by plotting the estimated marginal mean scores for each of the groups at each time point (Fig. 2), with a sharp increase in perceived control evident for the recovery group.

\subsection{Exploring the impact of control: Qualitative results}

Qualitative findings supported the quantitative analysis in demonstrating changes to perceived control. The thematic analysis also yielded deeper insights into the different ways in which control was impacted for people with EDs. The results show that control is more multifaceted than perhaps would be assumed from just the quantitative data; rather than just focusing upon a loss of control in the external environment, individuals reflected on increased control in other areas - primarily their eating and exercise behaviours.

Two key themes were generated: (1) ED behaviours as an 'auxiliary control mechanism'; (2) Loss of auxiliary control after lockdown. The
Table 2

Mean scores and standard deviation for each group at time point 1 (T1) and 2 (T2).

\begin{tabular}{|c|c|c|c|c|c|c|c|}
\hline \multirow[b]{2}{*}{ Score } & \multirow[b]{2}{*}{ Time } & \multicolumn{2}{|c|}{$\begin{array}{l}\text { Recovery }(\mathrm{n}= \\
11)\end{array}$} & \multicolumn{2}{|c|}{$\begin{array}{l}\text { Relapse }(\mathrm{n}= \\
9)\end{array}$} & \multicolumn{2}{|c|}{ Stable $(\mathrm{n}=38)$} \\
\hline & & M & SD & M & SD & M & SD \\
\hline \multirow[t]{2}{*}{ Wellbeing } & $\mathrm{T} 1$ & 17.08 & 3.30 & 17.59 & 1.74 & 16.49 & 3.28 \\
\hline & $\mathrm{T} 2$ & 18.58 & 1.89 & 16.92 & 2.11 & 16.76 & 3.51 \\
\hline \multirow[t]{2}{*}{ Perceived Stress } & $\mathrm{T} 1$ & 10.10 & 2.28 & 9.22 & 2.05 & 10.63 & 2.73 \\
\hline & $\mathrm{T} 2$ & 8.91 & 2.21 & 10.56 & 1.59 & 10.89 & 2.87 \\
\hline \multirow[t]{2}{*}{ Social Support } & $\mathrm{T} 1$ & 19.20 & 6.27 & 18.46 & 6.64 & 20.89 & 5.59 \\
\hline & $\mathrm{T} 2$ & 23.45 & 4.39 & 23.33 & 5.36 & 20.58 & 5.50 \\
\hline \multirow[t]{2}{*}{ Perceived Control } & $\mathrm{T} 1$ & 56.55 & 15.77 & 62.00 & 5.74 & 54.97 & 15.25 \\
\hline & $\mathrm{T} 2$ & 70.09 & 15.67 & 62.44 & 7.73 & 53.53 & 15.50 \\
\hline \multirow[t]{2}{*}{ ED Rumination } & $\mathrm{T} 1$ & 31.30 & 6.62 & 30.11 & 7.83 & 30.92 & 7.19 \\
\hline & T2 & 25.00 & 8.15 & 33.22 & 5.36 & 31.97 & 7.97 \\
\hline
\end{tabular}

development of these themes was informed by Froreich et al.'s (2016) observation that ED behaviours serve as an 'auxiliary control mechanism' when there is a perceived lack of control. The first theme reflects concerns around reduced control during lockdown, and the second focuses on reduced control after lockdown.

\subsection{Theme one: ED behaviours as an 'auxiliary control mechanism'}

Respondents reflected upon the unpredictable nature of the pandemic, and how this led to reduced feelings of control. Loss of general daily routine(s) was a major negative factor for many participants, an impact that has been felt by much of the population (Hou et al., 2020). This loss of external or 'general' control is likely to explain the quantitative differences in perceived control by the recovery group, versus the stable and relapse groups.

Lack of control over global situation has meant a bit more restrictive behaviours.

Some individuals reflected on their 'need' to establish new routines to regain a sense of control. However, this led to some respondents focusing their attention on controlling their eating behaviours - primarily as this represented an aspect of their life that they were able to control during these uncertain times:

I am more rigid about the times that I eat, this has only been since the pandemic began. I think it is because I need a new rigid routine as my old routines are missing that were not related to food or eating.

My eating disorder feels more valuable to me than ever. It is the only constant in what feels like a completely upside down and scary world, it is my only locus of control.

For these respondents, ED behaviours served as an important coping mechanism against a perceived loss of control in the external world, replacing this with a sense of control that was based on controlling their own behaviour. A similar discrepancy between desire for control and

Table 1

Means, Standard Deviations and Bivariate Correlations for each of the variables at each time point.

\begin{tabular}{|c|c|c|c|c|c|c|c|c|c|c|c|c|}
\hline Variable & M & SD & $1 \mathrm{a}$ & $1 b$ & $2 a$ & $2 b$ & $3 a$ & $3 b$ & $4 a$ & $4 \mathrm{~b}$ & $5 a$ & $5 b$ \\
\hline 1a. Wellbeing T1 & 16.78 & 3.05 & - & & & & & & & & & \\
\hline 1b. Wellbeing T2 & 16.81 & 3.83 & $.27^{*}$ & - & & & & & & & & \\
\hline 2a. Perc. Stress T1 & 10.26 & 2.59 & $-.65^{* * *}$ & -.21 & - & & & & & & & \\
\hline 2b. Perc. Stress T2 & 10.47 & 2.68 & $-.42 * *$ & $-.50 * * *$ & $.42^{* *}$ & - & & & & & & \\
\hline 3a. Soc. Support T1 & 20.98 & 5.86 & .17 & .06 & -.20 & .02 & - & & & & & \\
\hline 3b. Soc. Support T2 & 21.55 & 5.37 & -.12 & .10 & .01 & $-.26^{*}$ & $.49 * * *$ & - & & & & \\
\hline 4a. Perc. Control T1 & 56.36 & 14.34 & $.63^{* * *}$ & .08 & $-.56^{* * *}$ & $-.46^{* * *}$ & .18 & .07 & - & & & \\
\hline 4b. Perc. Control T2 & 58.05 & 15.87 & $.34 * *$ & $.35^{* * *}$ & $-.51 * * *$ & $-.79 * * *$ & .02 & $.33^{*}$ & $.50^{* * *}$ & - & & \\
\hline 5a. Rumination $\mathrm{T} 1$ & 30.76 & 7.03 & $-.42^{* *}$ & .08 & $.40^{* *}$ & .07 & -.11 & .25 & $-.47^{* *}$ & -.14 & - & \\
\hline 5b. Rumination $\mathrm{T} 2$ & 30.84 & 8.08 & -.18 & -.20 & .11 & $.37 * *$ & .14 & -.07 & -.15 & $-.36^{* *}$ & .24 & - \\
\hline
\end{tabular}

Note: ${ }^{*} \mathrm{p}<.05,{ }^{*} \mathrm{p}<.01, * * * \mathrm{p}<.001 . \mathrm{T} 1=$ time point 1 , April 2020, T2 = time point 2, June 2020. 


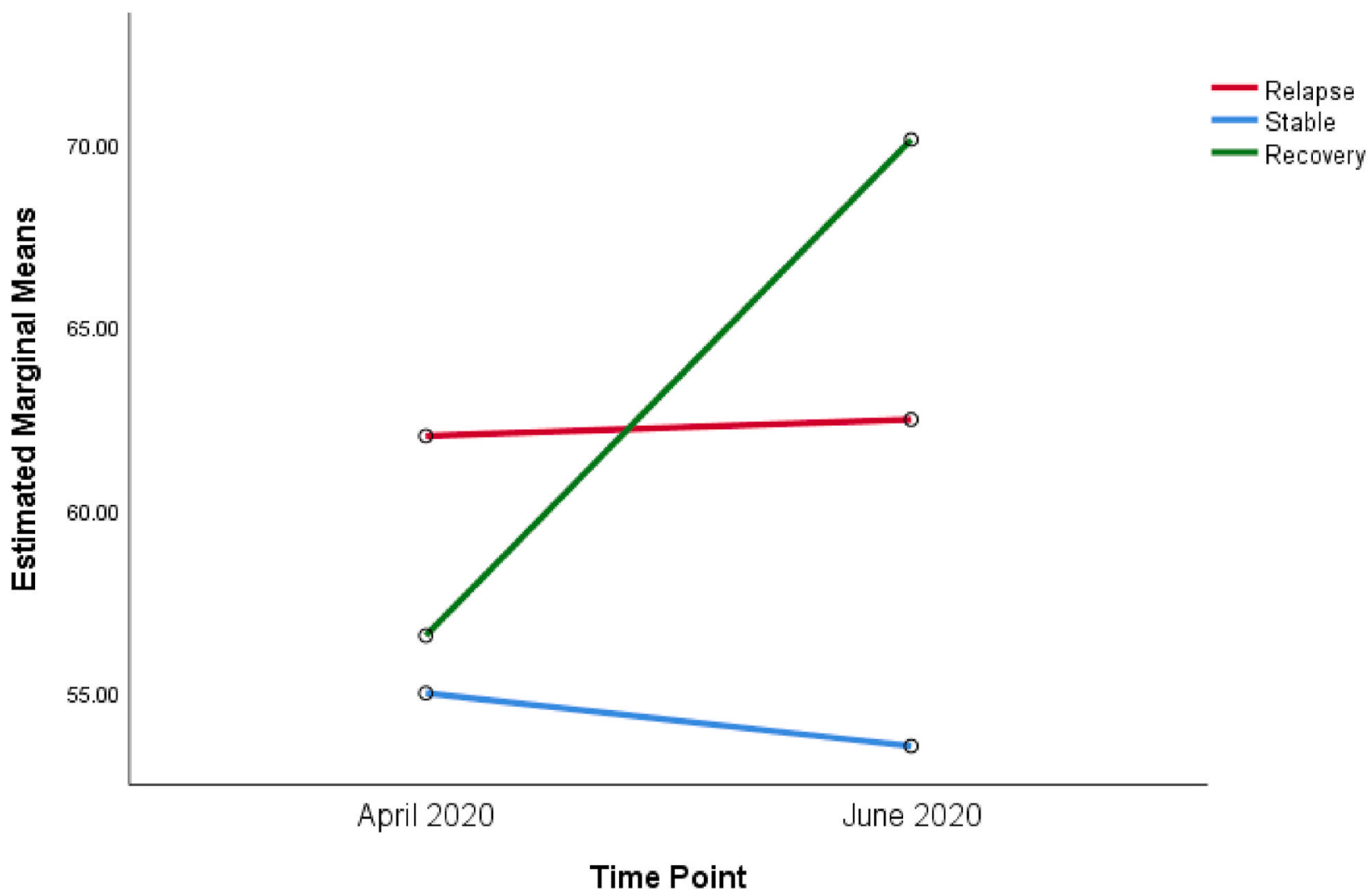

Fig. 2. Mean scores for perceived control at each time point, for each group (relapse, stable, and recovery).

perceived control has been shown to motivate Obsessive Compulsive Disorder (OCD) symptoms (Froreich et al., 2016).

Establishing increased control over their own eating and/or exercise behaviours was not as straight forward for some individuals, compared to others. In our initial survey, over one fifth of respondents reported changes to their living situation (Branley-Bell \& Talbot, 2020). For some this change meant increased isolation, but for many it involved suddenly finding themselves living with others. At follow-up, respondents living with others reported feeling less control over food purchases and meals, leading to stress, and worsened wellbeing:

Live with my parents so have found it quite stifling not having the space to eat how/where I would like.

Our findings suggest that ED symptoms can be driven by a discrepancy between respondents desire for control and low perceptions of actual control during the pandemic. ED behaviours can provide a means to (re-)establish some sense of control. However, further tensions can occur when an individual's control over their own behaviour is limited due to changes to living situations.

\subsection{Theme 2: Loss of auxiliary control after lockdown}

Our second theme focuses on reduced control after lockdown. However, again this relates to two sources of control: the wider control of the external world, and the narrower more self-focused control over eating and/or exercise behaviours. As aforementioned, whilst individuals felt that their general control had been negatively impacted due to the pandemic and lockdown, for many their control over their eating behaviours had increased. One respondent described having 'full and total control' over what they ate during the lockdown period:

I've been left to my own devices in isolation to have full and total control over what I eat.
However, respondents recognised that they would lose this element of control once lockdown measures eased. They described situations in which they would have reduced control and expressed concerns about their ability to cope, for example eating in social settings and/or having meals prepared by others:

I'm terrified at the thought of having to socialise over food again, and lose that aspect of total control I have over my life.

As the follow-up survey was completed when the first lockdown restrictions started to ease, respondents sometimes provided examples of situations when they felt a reduced sense of control:

I've found it harder now I can see friends as I have to deal with eating situations I've not been dealing with for a while e.g., picnics, where I am losing control which I had not been doing during lockdown. So I feel more guilt as I'm eating more and not losing weight.

In our initial survey, we found that going 'out' for food was an important factor in ED recovery that lockdown had impeded (Branley-Bell \& Talbot, 2020). Unfortunately, our findings suggest that people with EDs may face challenges transitioning from an environment in which they have increased control over their eating and/or exercise behaviours, to 'normality' where they typically have less control - even if this is a positive and necessary factor in their long-term ED recovery.

\section{Discussion}

In this study, we investigated the role of perceived control for people with experience of EDs during the early months of the COVID-19 pandemic. Our findings suggest that perceived control was a significant factor in ED recovery during this time, with higher perceptions of external control being associated with recovery. In contrast, those who experienced less perceived control reported relying on ED behaviours as an auxiliary coping mechanism. This presented challenges once the first 
lockdown restrictions started to ease, with some feeling anxious about relinquishing control over their eating habits.

Research around control as a factor in ED risk, maintenance and/or recovery can appear conflicting, particularly when considering quantitative findings. It has been suggested that this may be due to the multiplicity of constructs used to define control in the literature (e.g., sense of control, locus of control, mastery, need for perfectionism, and ineffectiveness). If studies are measuring different constructs of control, this will produce different results in relation to EDs, thus complicating our understanding of the link between ED and control (Froreich et al., 2016). Thankfully, most researchers are beginning to recognise that control is a multi-faceted, domain-specific factor (Froreich et al., 2016; Shapiro et al., 1993). Our results support the view that control should not be regarded, or referred to, as a singular factor. It is not sufficient to talk only of 'control' in relation to EDs, but to recognise that whilst individuals with ED symptoms may experience low perceptions of control in some areas (e.g., external control of their wider world/life), they may have high levels of control in others (e.g., internal control of their own behaviour). It is also important to recognise that some forms of control are healthy, whereas others can be debilitating.

Recently, Froreich et al. (2016) stated the importance of "determining which operationalisation of control is most centrally relevant to ED" (p.2). Our findings show that individuals experiencing ED recovery scored higher for general perceived control (measured by the SCI-general). However, those who did not experience improvement in their ED symptoms described other forms of control in their life - those that evolved around their eating and/or exercise behaviours. For these individuals, controlling their eating and exercise behaviours acted as a coping mechanism which allowed them to regain some sense of control. As described by Froreich et al. (2016) "In the absence of adaptive personal control strategies, the individual may be driven to enact ritualistic body control as an auxiliary control mechanism" (p. 2). Our research further emphasises the importance of distinguishing between different types of control when conducting ED-related research and/or when designing interventions.

Specific measures for future research will rely heavily upon the specific research question(s) and aim(s). However, we would like to note that consideration should not be limited solely to whether researchers wish to measure internal and/or external control; but that they must also pay careful consideration to the level of precision of the measures. In our study we differentiate between two broad domains of control - external/ general and internal. However, there are likely to be deeper levels within each of these categories. For example, internal control may contain many domains such as control over the body, the mind, emotions, etc. Whilst individuals currently experiencing an $\mathrm{ED}(\mathrm{s})$ appear to demonstrate high levels of control over their body in relation to eating and exercise behaviour, it is possible that they may score differently across other internal domains. Likewise, there are likely to be many different domains in relation to external control (e.g., work, family, the wider world). While for our research, these two overarching categories were appropriate, future researchers should reflect on their research aims in relation to whether a greater level of precision or specificity is required.

Previously research exploring the role of control for individuals with EDs has tended to be restricted to patients diagnosed with anorexia nervosa (Froreich et al., 2016). One of the strengths of our study is that we included individuals with a range of different EDs, suggesting that control may be a relevant factor across EDs more widely. A further strength of this research is that we were able to collect data at two landmark moments during the pandemic: Firstly, the start of the pandemic within the UK and the introduction of the first national lockdown, and secondly, the easing of the first lockdown restrictions. This enabled us to explore respondents' experiences as they transitioned into this new way of living, and again as they faced their first transition out of lockdown; providing unique insight into the impact this unprecedented event has had for individuals experiencing EDs.
An interesting avenue for further research would be to compare our findings from the early stages of the pandemic, with later stages. The pandemic has now been prolonged in nature and multiple lockdown restrictions have been imposed, eased and reintroduced. There have also been reports of confusion and apprehension over many aspects of the situation, including what new and changing restrictions mean (UCL News, 2020a), when the pandemic will be under control (Charumilind et al., 2020), the rising UK death toll (Sandle, 2021) and general handling of the situation (Kings College London News, 2020). All these factors are likely to further impact upon perceived control. However, interestingly some early research suggests that mental wellbeing may have been most negatively impacted at the start of the pandemic (UCL News, 2020b). Further research tracking wellbeing and identifying coping mechanisms could provide valuable insight into how individuals with EDs are coping with the pandemic; with implications for treatment and preparation for similar occurrences in the future.

Despite the identified strengths, our study does have some limitations. Firstly, we relied on self-reports of ED status, rather than operationalised measures. We opted for self-identification to promote inclusivity, as it is debated whether psychometric measures are accurate enough to suitably capture the range of different EDs (e.g., Burton et al., 2016) and whether they can potentially introduce bias (for example towards female-specific ED symptomology, Dahlgren et al., 2018). There is research to suggest that self-reported ED diagnoses using simple screening questions - although not perfect - can be at least as good as other available psychometric instruments (e.g., Keski-Rahkonen et al., 2006). However, whilst we value self-identification, we recognise that omitting an operationalised measure limits the ability to compare our results with other studies (perhaps particularly for recovery status as individuals' perceptions of recovery or relapse may differ from clinically assessed definitions). We suggest that future research looking to build upon these preliminary findings considers incorporating a suitable psychometric measure.

Due to the sudden and unexpected onset of the pandemic, it was also not possible to obtain measures prior to the pandemic. Consequently, we cannot examine whether the lockdown measures were associated with changes in the quantitative measures. In future, data from large cohort studies could be analysed to determine the impact of the pandemic on ED severity and different types of EDs.

Moreover, we used a survey approach to reach a wide range of people with EDs; however, this sometimes resulted in qualitative responses being quite brief. In future, we recommend researchers conduct in-depth interviews with this population to gain deeper insight into control and the role it played during the pandemic. Finally, our sample size for the recovery and relapse groups were relatively small, and as with any longitudinal study there is the potential that dropout rates may have introduced selection bias into the data (for example if the individuals who chose not to participate at the second time point differ systematically in some way from those who did participate). Therefore we wish to highlight the preliminary nature of the findings and recommend further research into the impact of control on relapse and recovery using larger samples.

Another interesting avenue for future research involves exploring whether people with different eating disorders experience control in different ways. For example, Zysberg and Tell (2013) found nonlinear associations with anorexia nervosa, whereby low and high control corresponded with higher anorexia nervosa scores; whereas, a positive relationship was found between bulimia nervosa symptoms and perceived control. Although it is beyond the scope of our analysis, future researchers could investigate between-group differences across ED type.

Our findings suggest that perceived control may play an important role in ED recovery, this has implications for future ED prevention, treatment, and assessment. For instance, should future research support our findings in larger, more diverse samples, then future healthcare interventions may wish to introduce, or strengthen, aspects of treatment based upon increasing individuals' general sense of control in their life 
and the external environment. An example of this may be in cognitive behavioural therapies, which traditionally aim to assess and modify maladaptive beliefs. Biased beliefs in control are a prevalent cognitive distortion among people with EDs, particularly anorexia nervosa (Sassaroli et al., 2008). Our preliminary findings suggest that there may be value in therapies that aim to challenge beliefs about control in different domains.

Government bodies may also wish to consider the impact that wording around the pandemic could have upon this population (Talbot \& Branley-Bell, 2021). For example, public health campaigns may be more beneficial and/or effective if they emphasise ways in which individuals can (re-)gain a sense of control over the situation.

\section{Conclusions}

In conclusion, our findings demonstrate that perceived control may be an important - albeit complex - factor in the aetiology of EDs. High levels of general control appear to be beneficial and associated with ED recovery. When general control of the external environment is low, individuals may rely on more harmful coping mechanisms around controlling their own eating and/or exercise behaviours. Implications for future research (within the field of ED research and more widely) include the recognition of the multifaceted nature of control, and that the impact of control - whether beneficial or debilitating - may depend upon the specific domain and circumstances.

\section{Author contributions}

DB managed the project. Both authors conceived the study design and oversaw data collection. DB conducted the quantitative analysis; CT conducted the qualitative analysis. Both authors agreed on the themes generated by thematic analysis. DB prepared the manuscript draft, both authors edited and approved the final submitted manuscript.

\section{Data availability}

Upon request to the corresponding author.

\section{Funding}

This research did not receive any specific grant from funding agencies in the public, commercial, or not-for-profit sectors.

\section{Ethics approval and consent to participate}

Ethical approval was granted by Northumbria University ethics committee [23259] prior to data collection and all participants provided informed consent.

\section{Appendix A. Supplementary data}

Supplementary data to this article can be found online at https://doi. org/10.1016/j.appet.2021.105596.

\section{References}

Branley-Bell, D., \& Talbot, C. V. (2020). Exploring the impact of the COVID-19 pandemic and UK lockdown on individuals with experience of eating disorders. Journal of Eating Disorders, 8(1). https://doi.org/10.1186/s40337-020-00319-y, 44-44.

Braun, V., \& Clarke, V. (2006). Using thematic analysis in psychology. Qualitative Research in Psychology, 3(2), 77-101. https://doi.org/10.1191/1478088706qp063oa Bruch, H. (2001). The golden cage: The enigma of anorexia nervosa. Harvard University Press.

Burton, A. L., Abbott, M. J., Modini, M., \& Touyz, S. (2016). Psychometric evaluation of self-report measures of binge-eating symptoms and related psychopathology: A systematic review of the literature. International Journal of Eating Disorders, 49(2), 123-140. https://doi.org/10.1002/eat.22453

Charumilind, S., Craven, M., Lamb, J., Sabow, A., \& Wilson, M. (2020). When will the COVID-19 pandemic end? McKinsey. McKinsey \& Company Healthcare Systems \&
Services. https://www.mckinsey.com/industries/health care-systems-and-services/our-insights/when-will-the-covid-19-pandemic-end\#.

Cohen, S., Kamarck, T., \& Mermelstein, R. (1983). A global measure of perceived stress. Journal of Health and Social Behavior, 24.

Cowdrey, F. A., \& Park, R. J. (2012). The role of experiential avoidance, rumination and mindfulness in eating disorders. Eating Behaviors, 13(2), 100-105. https://doi.org/ 10.1016/j.eatbeh.2012.01.001

Dahlgren, C. L., Stedal, K., \& Wisting, L. (2018). A systematic review of eating disorder prevalence in the nordic countries: 1994-2016. Nordic Psychology, 70(3), 209-227. https://doi.org/10.1080/19012276.2017.1410071

Dalgleish, T., Tchanturia, K., Serpell, L., Hems, S., De Silva, P., \& Treasure, J. (2001). Perceived control over events in the world in patients with eating disorders: A preliminary study. Personality and Individual Differences, 31(3), 453-460. https://doi. org/10.1016/S0191-8869(00)00150-1

Doll, H. A., Petersen, S. E., \& Stewart-Brown, S. L. (2005). Eating disorders and emotional and physical well-being: Associations between student self-reports of eating disorders and quality of life as measured by the SF-36. Quality of Life Research, 14(3), 705-717. https://doi.org/10.1007/s11136-004-0792-0

Fairburn, C. G., Shafran, R., \& Cooper, Z. (1999). A cognitive behavioural theory of anorexia nervosa. Behaviour Research and Therapy, 37(1), 1-13. https://doi.org/ 10.1016/S0005-7967(98)00102-8

Froreich, F. V., Vartanian, L. R., Grisham, J. R., \& Touyz, S. W. (2016). Dimensions of control and their relation to disordered eating behaviours and obsessive-compulsive symptoms. Journal of Eating Disorders, 4(1), 14. https://doi.org/10.1186/s40337016-0104-4

Ghaderi, A. (2003). Structural modeling analysis of prospective risk factors for eating disorder. Eating Behaviors, 3(4), 387-396. https://doi.org/10.1016/S1471-0153(02) 00089-2

Hempel, R., Vanderbleek, E., \& Lynch, T. R. (2018). Radically open DBT: Targeting emotional loneliness in anorexia nervosa. Eating Disorders, 26(1), 92-104. https:// doi.org/10.1080/10640266.2018.1418268

Hou, W. K., Lai, F. T., Ben-Ezra, M., \& Goodwin, R. (2020). Regularizing daily routines for mental health during and after the COVID-19 pandemic. Journal of Global Health, 10(2). https://doi.org/10.7189/jogh.10.020315

Hou, F., Xu, S., Zhao, Y., Lu, Q., Zhang, S., Zu, P., Sun, Y., Su, P., \& Tao, F. (2013). Effects of emotional symptoms and life stress on eating behaviors among adolescents. Appetite, 68, 63-68. https://doi.org/10.1016/j.appet.2013.04.010

Keski-Rahkonen, A., Sihvola, E., Raevuori, A., Kaukoranta, J., Bulik, C. M., Hoek, H. W., Rissanen, A., \& Kaprio, J. (2006). Reliability of self-reported eating disorders: Optimizing population screening. International Journal of Eating Disorders, 39(8), 754-762. https://doi.org/10.1002/eat.20277

Kings College London News. (2020). Majority of Britons distrust government Covid response for first time. https://www.kcl.ac.uk/news/majority-of-britons-distrust-go vernment-covid-response-for-first-time.

Lee, S., Chan, L., Kwok, K., \& Hsu, L. K. G. (2005). Relationship between control and the intermediate term outcome of anorexia nervosa in Hong Kong-, 2005. Australian and New Zealand Journal of Psychiatry, 39(3), 141-145. https://doi.org/10.1080/ j.1440-1614.2005.01535.x

Marcos, Y., \& Cantero, M. C. (2009). Assesment of social support dimensions in patients with eating disorders. Spanish Journal of Psychology, 12, 226-235. https://doi.org/ 10.1017/S1138741600001633

McCombie, C., Austin, A., Dalton, B., Lawrence, V., \& Schmidt, U. (2020). "Now it's just old habits and misery"-understanding the impact of the covid-19 pandemic on people with current or life-time eating disorders: A qualitative study. Frontiers in Psychiatry, 11. https://doi.org/10.3389/fpsyt.2020.589225

Mitchell, A. M., Crane, P. A., \& Kim, Y. (2008). Perceived stress in survivors of suicide: Psychometric properties of the perceived stress scale. Research in Nursing \& Health, 31(6), 576-585. https://doi.org/10.1002/nur.20284

Nhs Health Scotland. (2016). Warwick-edinburgh mental well-being scale (WEMWBS) user guide.

Park, R. J., Dunn, B. D., \& Barnard, P. J. (2011). Schematic models and modes of mind in anorexia nervosa I: A novel process account. International Journal of Cognitive Therapy, 4(4), 415-437. https://doi.org/10.1521/ijct.2011.4.4.415

Pfefferbaum, B., \& North, C. S. (2020). Mental health and the covid-19 pandemic. New England Journal of Medicine, 383(6), 510-512. https://doi.org/10.1056/ NEJMp2008017

Phillipou, A., Meyer, D., Neill, E., Tan, E. J., Toh, W. L., Van Rheenen, T. E., \& Rossell, S. L. (2020). Eating and exercise behaviors in eating disorders and the general population during the COVID-19 pandemic in Australia: Initial results from the COLLATE project. International Journal of Eating Disorders, 53(7), 1158-1165. https://doi.org/10.1002/eat.23317

Rawal, A., Park, R. J., \& Williams, J. M. G. (2010). Rumination, experiential avoidance, and dysfunctional thinking in eating disorders. Behaviour Research and Therapy, 48 (9), 851-859. https://doi.org/10.1016/j.brat.2010.05.009

Richardson, C., Patton, M., Phillips, S., \& Paslakis, G. (2020). The impact of the COVID19 pandemic on help-seeking behaviors in individuals suffering from eating disorders and their caregivers. General Hospital Psychiatry, 67, 136-140. https://doi. org/10.1016/j.genhosppsych.2020.10.006

Rodgers, R. F., Lombardo, C., Cerolini, S., Franko, D. L., Omori, M., FullerTyszkiewicz, M., Linardon, J., Courtet, P., \& Guillaume, S. (2020). The impact of the COVID-19 pandemic on eating disorder risk and symptoms. International Journal of Eating Disorders, 53(7), 1166-1170. https://doi.org/10.1002/eat.23318

Rodríguez-Rey, R. (2020). Psychological impact of COVID-19 in Spain: Early data report Psychological trauma: Theory, research, practice, and policy; US. Educational Publishing Foundation. https://doi.org/10.1037/tra0000943 
Sandle, A. M., \& Paul. (2021, January 25). Anger and grief as United Kingdom's COVID-19 death toll nears 100,000. Reuters. https://www.reuters.com/article/us-health-cor onavirus-britain-deaths-idUSKBN29U13M.

Sassaroli, S., Gallucci, M., \& Ruggiero, G. M. (2008). Low perception of control as a cognitive factor of eating disorders. Its independent effects on measures of eating disorders and its interactive effects with perfectionism and self-esteem. Journal of Behavior Therapy and Experimental Psychiatry, 39(4), 467-488. https://doi.org/ 10.1016/j.jbtep.2007.11.005

Schlegl, S., Maier, J., Meule, A., \& Voderholzer, U. (2020). Eating disorders in times of the COVID-19 pandemic - results from an online survey of patients with anorexia nervosa. International Journal of Eating Disorders, 53(11), 1791-1800. https://doi. org/10.1002/eat.23374

Shapiro, D. H., Blinder, B. J., Hagman, J., \& Pituck, S. (1993). A psychological 'sense-ofcontrol' profile of patients with anorexia nervosa and bulimia nervosa. Psychological Reports, 73(2), 531-541. https://doi.org/10.2466/pr0.1993.73.2.531

Shapiro, D. H., Jr. (1994). Shapiro control inventory (SCI) manual. http://controlresear ch.net/shapiro-control-inventory-manual.html.

Smith, K. E., Mason, T. B., \& Lavender, J. M. (2018). Rumination and eating disorder psychopathology: A meta-analysis. In Clinical psychology review (Vol. 61, pp. 9-23). Elsevier Inc. https://doi.org/10.1016/j.cpr.2018.03.004.

Talbot, C. V., \& Branley-Bell, D. (2021). \#BetterHealth: A qualitative analysis of reactions to the UK government's better health campaign. Journal of Health Psychology. https://doi.org/10.1177/1359105320985576, 1359105320985576.

Tennant, R., Hiller, L., Fishwick, R., Platt, S., Joseph, S., Weich, S., Parkinson, J., Secker, J., \& Stewart-Brown, S. (2007). The warwick-edinburgh mental well-being scale (WEMWBS): Development and UK validation. Health and Quality of Life Outcomes, 5(1), 1-13. https://doi.org/10.1186/1477-7525-5-63

Termorshuizen, J. D., Watson, H. J., Thornton, L. M., Borg, S., Flatt, R. E., Macdermod, C. M., Harper, L. E., Van Furth, E. F., Peat, C. M., \& Bulik, C. M. (2020). Early impact of COVID-19 on individuals with eating disorders: A survey of $\sim 1000$ individuals in the United States and The Netherlands. MedRxiv. https://doi.org/ 10.1101/2020.05.28.20116301, 2020.05.28.20116301-2020.05.28.20116301.

Ucl News. (2020, October 23). Half of public don't 'fully understand' current Covid-19 lockdown rules. UCL News. https://www.ucl.ac.uk/news/2020/oct/half-public-dont -fully-understand-current-covid-19-lockdown-rules.

Ucl News. (2020, December 10). 'Psychological toll' of lockdown peaked in the early weeks. UCL News. https://www.ucl.ac.uk/news/2020/dec/psychological-toll-lockdown -peaked-early-weeks.

Usher, K., Durkin, J., \& Bhullar, N. (2020). The COVID-19 pandemic and mental health impacts. International Journal of Mental Health Nursing, 29(3), 315-318. https://doi. org/10.1111/inm.12726

Vaglio, J., Conard, M., Poston, W. S., O’Keefe, J., Haddock, C. K., House, J., \& Spertus, J. A. (2004). Testing the performance of the ENRICHD social support instrument in cardiac patients. Health and Quality of Life Outcomes, 2, 24. https://doi. org/10.1186/1477-7525-2-24

Vallejo, M. A., Vallejo-Slocker, L., Fernández-Abascal, E. G., \& Mañanes, G. (2018), Determining factors for stress perception assessed with the perceived stress scale (PSS-4) in Spanish and other European samples. Frontiers in Psychology, 9(JAN), 37. https://doi.org/10.3389/fpsyg.2018.00037

Vuillier, L., May, L., Greville-Harris, M., Surman, R., \& Moseley, R. L. (2021). The impact of the COVID-19 pandemic on individuals with eating disorders: The role of emotion regulation and exploration of online treatment experiences. Journal of Eating Disorders, 9(1), 10. https://doi.org/10.1186/s40337-020-00362-9

Yao, H., Chen, J. H., \& Xu, Y. F. (2020). Patients with mental health disorders in the COVID-19 epidemic. The Lancet Psychiatry, 7(4). https://doi.org/10.1016/S22150366(20)30090-0. e21-e21.

Zheng, L., Miao, M., \& Gan, Y. (2020). Perceived control buffers the effects of the COVID19 pandemic on general health and life satisfaction: The mediating role of psychological distance. Applied Psychology. Health and Well-Being, 12(4), 1095-1114.

Zysberg, L., \& Tell, E. (2013). Emotional intelligence, perceived control, and eating disorders. SAGE Open, 3(3). https://doi.org/10.1177/2158244013500285 